\title{
The Impact of the Medicare Part D Prescription Benefit on Generic Drug Use
}

\author{
James X. Zhang, $P h D^{7}$, Wesley Yin, $P h D^{2,3,4}$, Shawn X. Sun, $P h D^{5}$, \\ and $G$. Caleb Alexander, $M D, M S^{2,6,7,8}$
}

'Department of Pharmacy, School of Pharmacy, Virginia Commonwealth University, Richmond, VA, USA; ${ }^{2}$ Center for Health and Social Sciences, University of Chicago, Chicago, IL, USA; ${ }^{3}$ Harvard University, Robert Wood Johnson Scholars Program in Health Policy, Cambridge, MA, USA; ${ }^{4}$ University of Chicago, Harris School of Public Policy, Chicago, IL, USA; ${ }^{5}$ Health Outcomes Department, Walgreens Health Services, Deerfield, IL, USA; ${ }^{6}$ Section of General Internal Medicine, Department of Medicine, University of Chicago, Chicago, IL, USA; ${ }^{7}$ MacLean Center for Clinical Medical Ethics, University of Chicago, Chicago, IL, USA; ${ }^{8}$ Department of Pharmacy Practice, University of Illinois at Chicago School of Pharmacy, Chicago, IL, USA.

BACKGROUND: Little information exists regarding the impact of Medicare Part D on generic drug use.

OBJECTIVE: To examine changes in the use of generic prescriptions attributable to Part D among a sample of Medicare beneficiaries.

DESIGN, PARTICIPANTS, AND MEASUREMENTS: Difference-in-difference analysis of pharmacy claims of Part D enrollees and non-enrollees aged 67-79 years from 2005 to 2006. The final sample represented approximately 2.4 million unique subjects. Analyses were conducted separately for major therapeutic classes, limited to subjects filling at least one prescription within the class during 2005 and 2006, and adjusted for subject characteristics, prescription characteristics, socio-demographic characteristics measured through zipcode-linked Census data, baseline differences between Part D and non-Part D enrollees, and secular trends in generic use.

RESULTS: Generic drugs accounted for $58 \%$ of total prescriptions. Among the entire group of beneficiaries, there was a trend of increased generic drug use in 13 out of 15 drug classes examined. However, after adjusting for potential confounders, the growth rate of generic drug use was lower among Part D enrollees than among non-enrollees; enrollees were slightly less likely to fill prescriptions for generic drugs vs. brand-name drugs in 2006 compared to 2005 (odds ratio 0.95, 95\% confidence interval 0.94-0.95).

CONCLUSIONS: Despite secular trends of increased utilization of generic drugs among both Part D enrollees and non-enrollees, the net impact of Part D among these beneficiaries was a modest decrease in the use of generic drugs. This finding, which is consistent with economic theory but contrary to several recent reports, highlights the complexity of assessing the impact of Part D on overall consumer welfare.

Received January 22, 2008

Revised June 11, 2008

Accepted July 8, 2008

Published online July 26, 2008
KEY WORDS: medicare Part D; prescription coverage;

pharmacoepidemiology; generic drugs.

J Gen Intern Med 23(10): 1673-8

DOI: $10.1007 / \mathrm{s} 11606-008-0742-6$

(c) Society of General Internal Medicine 2008

\section{INTRODUCTION}

The Medicare Modernization Act Prescription Drug Benefit (Part D) represents a broad change in pharmaceutical policy for tens of millions of Medicare beneficiaries ${ }^{1}$. Early studies of the program have focused on its uptake $^{2}$ and beneficiary reaction to the benefit ${ }^{3}$, while other reports have examined how the benefit has impacted prescription drug expenditures among those who have enrolled in Prescription Drug Plans $(\mathrm{PDPs})^{4}$. However, few studies have been performed examining the impact of the benefit on prescription drug utilization among a broad population of Part D enrollees and nonenrollees alike.

Projections of the impact of Part D, based on estimates of how demand for drugs changes as a function of cost-sharing and the number of people who already had prescription drug insurance prior to 2006, suggested that the benefit would lead to small increases in overall utilization and somewhat larger decreases in out-of-pocket expenditures ${ }^{5}$. Previously, we examined this among a large sample of beneficiaries including Part D enrollees and non-enrollees and found an approximate $6 \%$ increase in utilization and $13 \%$ decrease in out-of-pocket costs from 2005 to $2006^{6}$. However, despite expanded access to prescription drugs as a result of Part D, few studies have isolated the impact of Part D on generic drug use. Rather, reports that have been released, such as an analysis of generic drug use in Part D conducted by the Department of Health and Human Services, have tended to examine rates of generic use among Part D enrollees alone ${ }^{7}$.

Assessing the impact of Part D on generic use is important because increasing generic utilization has been considered a key policy tool to save consumers and society tens of billions of dollars $^{8,9}$; the average cost of generic drugs is $71 \%$ less than their brand name equivalents ${ }^{10}$. Although generic drug use now accounts for $63 \%$ of all US prescription drugs, considerably greater rates of generic utilization are possible. 
It is not clear what the ultimate impact of Part $\mathrm{D}$ on generic drug use will be, since there are plausible mechanisms whereby Part D may lead to both increases and decreases in generic drug use. On the one hand, given that Part D subsidizes the cost of prescription drugs, economic theory would suggest that this would tend to increase the use of more expensive, brand-name drugs relative to their less expensive generic counterparts; increasing coverage allows seniors to utilize expensive branded drugs at significantly lower outof-pocket costs than they would have under no insurance or under insurance prior to Part D implementation. Such increases might also be supported by widespread beliefs that generic drugs are inferior to those that are brand named ${ }^{11}$. On the other hand, there have been several efforts to limit drug manufacturers from artificially extending expiring patents ${ }^{12}$, thereby potentially increasing the number of generic entrants for key blockbuster drugs. In addition, tiered Part D formularies and the "doughnut hole" included in many plans (a gap in the standard Part D benefit during which there is no thirdparty prescription coverage) may have further sensitized consumers to prescription costs and contributed to increased rates of generic use, consistent with publicized anecdotal and media reports ${ }^{13-15}$. Observations that costs associated with Part D have not increased as much as expected ${ }^{16}$ also raise the question of the role that unexpected increases in utilization of generic drugs over branded counterparts could play, rather than over-estimates of the growth rate of drug prices or other causes.

In addition to the impact of Part D on generic drug use, we were also interested in examining the potential cost implications of greater generic substitution among Part D enrollees. We undertook these analyses using data from a national pharmacy chain that accounts for approximately 15\% of prescription drugs dispensed in the United States.

\section{METHODS}

\section{Data}

We selected a $5 \%$ random sample of unique pharmacy customers who filled at least one prescription between January 1, 2005 and December 31, 2006 at any retail or mail-order member of the pharmacy chain. For each of these subjects, we obtained claims data for every prescription filled between January 1, 2005 and December 31, 2006. Thus, these claims represent 12 months of observation prior to and following January 1, 2006, the date of Part D implementation.

For each prescription claim, we obtained data including subjects' demographic characteristics (age, sex, language preference, zip code of residence), insurance characteristics (prescription drug plan, method of payment), pharmacy characteristics (zip code location), and prescription characteristics [National Drug Code (NDC), therapeutic class, drug dose, number of treatment days, date dispensed, number of refills). We were able to identify dual-eligible subjects based on their having filled claims paid by Medicaid 2005, given knowledge that the turnover or "churning" of Medicaid subjects in this age range is quite low ${ }^{17}$. However, our data did not allow for us to discern between subjects enrolled in Prescription Drug Plans (PDPs) or Medicare Advantage Plans (MAPDs). We used data on subjects' zip code of residence (i.e., the residence recorded at subjects' first pharmacy claim in 2005) to link the pharmacy claims data to data from the 2000 Census, including information on the total population, median household income, income per capita, fraction urban, fraction African American, unemployment rate, and poverty rate within the zip code of residence ${ }^{18,19}$.

Previously, we used data from the Behavioral Risk Factor Surveillance System (BRFSS) to compare the characteristics of our subjects with those of all Medicare beneficiaries. These analyses indicated that our subjects were much more likely to reside in urban areas than a nationally representative beneficiary sample, but were otherwise similar to the broader Medicare population ${ }^{6}$.

\section{Analyses}

We used a differences-in-differences strategy ${ }^{20}$ to estimate the impact of the Part D drug benefit for subjects aged 67-79. This approach compares the pre-post difference in generic drug use of a control population with the pre-post difference in generic drug use of the subjects of interest, those enrolled in Part D plans. Thus, the difference-in-differences strategy allows one to identify the effect attributable to Part D after accounting for any possible secular trend. We excluded subjects age 80 and over, since the proportion of subjects in nursing home care is higher, and although they may be covered through the Medicare Modernization Act, changes in coverage regarding nursing and long-term care subjects ${ }^{21}$ do not extend to the majority of the Medicare population. We chose age 67 as the age cut-off for inclusion rather than 66 because we wanted to avoid including subjects in our analyses who had less than a full year of Medicare eligibility during the pre-period (2005). We conducted separate analyses for each therapeutic class. In our primary analysis, for each drug class, we defined our intervention group as subjects ages of 67-79 who enrolled in the Part D drug program and had at least one prescription in 2005 (prior to the institution of Part D) and at least one prescription in 2006 (after the institution of Part D) within that class. Such a strategy helped to ensure that observations regarding changes in utilization were not due to an unbalanced patient panel caused by uneven entry into or attrition from the panel $^{22}$. In other words, beneficiaries may die, move, or otherwise switch to another pharmacy chain, and thus our strategy was aimed at increasing the proportion of subjects who remained customers at the pharmacy during the study period. In our primary analyses, we used a patient perspective on Medicare Part D and therefore defined our control group as beneficiaries ages 67-79 that did not enroll in the benefit. We also conducted analyses where we defined our control group as subjects who were ages 60-63 as of January 1, 2006, as these subjects were most similar to Medicare beneficiaries yet not eligible for the benefit during any point during 2005 and 2006. We included indicator variables reflecting Part D enrollment (reflecting baseline differences between treatment and control group), the year of observation (2005 vs. 2006), and an interaction term between these two variables (representing the effect of Part D net baseline differences between enrollees and non-enrollees and net time trends).

We defined our primary outcome variable as generic drug use and used multivariate logistic regression to examine the association between predictors of interest and this outcome variable. The unit of analysis in the logistic regression was a 
single claim. We included covariates in our analyses including beneficiaries' age, gender, English language preference (derived from the pharmacy claims), socio-demographic characteristics measured through zip code-linked Census data, the number of pharmacy claims, and Medicaid eligibility in the base year. We also included measures of subjects' generosity of prescription insurance and comorbid burden.

Our measure of generosity of drug insurance plans was a ratio of out-of-pocket payments to total drug costs. However, measurement of generosity level of outpatient prescription drug coverage has been developed based upon a single insurance $\operatorname{plan}^{23}$. Since beneficiaries can have multiple plans during a given year, a measure of generosity of a single plan may not sufficiently reflect the beneficiaries' experiences and financial risks during the year. Therefore, we defined a "payout ratio" that summed out-of-pocket payments across claims, and plans across years, and that was then divided by the total payments (out-of-pocket plus plan payments). Subjects who fill very few prescriptions may not receive any reimbursements from their insurance plan and thus may have an artificially high payout ratio that doesn't reflect the true generosity of their prescription coverage. Therefore, we also adjusted for the number of claims in the baseline year in the regression models.

We used the Chronic Disease Score (CDS) to control for subjects' comorbid conditions. This method of risk stratification, which is derived from patients' age, gender, and NDCs reported in the pharmacy claims, has been validated as a predictor of physician-rated disease status, self-reported health, risk of hospitalization, and death ${ }^{24}$. Based upon CDS, we created 29 indicator variables for the corresponding disease categories during our baseline year (2005).

In our analyses, we focused on 15 therapeutic classes (Table 2). We selected nine of these because they are among the most commonly used drug classes; together, they account for approximately half of all prescriptions dispensed annually in the United States based on nationally representative data for the entire US population ${ }^{25}$. Of these classes, our classification of ACE-inhibitors excluded angiotensin-receptor blockers and our classification of anti-ulcerants included both proton pump inhibitors as well as histamine-2 antagonists. We included two classes (barbiturates and benzodiazepines) because the Medicare Modernization Act (MMA) forbids Prescription Drug Plans (PDPs) from including them within their formularies, and four others classes (antipsychotics, anticonvulsants, antiretrovirals, and antineoplastics) because the MMA made special provisions for coverage of "all or substantially all" members of these drug classes. There is not one agreed-upon taxonomic organization of prescription drugs; we used classifications based upon a taxonomy provided by First Data Bank, a vendor that provides pharmaceutical software widely used in the health-care industry. All analyses were conducted using STATA Version 9 (Stata Corp, College Station, TX, 2006).

\section{RESULTS}

\section{Subject Characteristics}

Table 1 describes subjects' characteristics. Overall, our sample included 117,970 subjects ages $67-79$, representing 2.4 million Medicare beneficiaries. Among these subjects, 31\% had enrolled in Part D as of Dec 31, 2006. Table 1 also depicts subjects' demographic characteristics and characteristics of their prescription coverage and comorbid conditions. On average, Part D enrollees appeared to have more pharmacy claims, a higher out-of-pocket payout ratio, and more chronic diseases than Part D non-enrollees.

\section{Aggregate Changes in Utilization}

Table 2 depicts the change of rates in generic drug use of each drug class of interest without adjustment for patient characteristics, the impact of Part D, and secular trends. For example, in 2005, about 56 percent of all prescriptions were generic, and the rate increased to 58 percent in 2006. However, the change in rates of generic drug utilization was not uniform across therapeutic classes. For example, while there were large increases in generic use of antihyperlipidemics and antihistamines, there were also therapeutic classes with decreases in generic drug use, such as beta-blockers and anticonvulsants. There was general concordance in the

Table 1. Description of Subjects Filling Prescriptions within a National Pharmacy Chain

\begin{tabular}{|c|c|c|c|}
\hline & \multicolumn{3}{|c|}{ Subjects ages $67-79(n=117,970)$} \\
\hline & Medicare Part D enrollees & Non-enrollees & P-value \\
\hline $\mathrm{N}(\%)$ & $36,833(31.2)$ & $81,137(68.8)$ & \\
\hline Age, years (SE) & $72.5(3.72)$ & $72.3(3.74)$ & $<0.01$ \\
\hline Female sex, percent & 63.2 & 55.3 & $<0.01$ \\
\hline English language preference, percent & 93.0 & 96.0 & $<0.01$ \\
\hline \multicolumn{4}{|l|}{ Characteristics of zip-code based on 2000 Census data } \\
\hline Total population, thousands (SE) & $31.1(17.7)$ & $29.3(16.4)$ & $<0.01$ \\
\hline Median household income, thousands (SE) & $45.0(16.7)$ & $46.2(16.5)$ & $<0.01$ \\
\hline Income per capita, thousands (SE) & $22.8(10.0)$ & $23.5(9.60)$ & $<0.01$ \\
\hline Fraction urban population, percent (SE) & $90.5(21.5)$ & $88.9(23.0)$ & $<0.01$ \\
\hline Fraction African-American, percent (SE) & $12.9(21.1)$ & $11.7(19.2)$ & $<0.01$ \\
\hline Employment rate, percent (SE) & $94.1(3.81)$ & $94.6(3.28)$ & $<0.01$ \\
\hline Poverty rate, percent $(\mathrm{SE})$ & $12.0(8.80)$ & $10.6(7.71)$ & $<0.01$ \\
\hline Total number of prescriptions dispensed in 2005 (SE) & $29.8(29.3)$ & $20.9(25.0)$ & $<0.01$ \\
\hline Out-of-pocket payout ratio in 2005 (SE) & $0.56(0.40)$ & $0.48(0.36)$ & $<0.01$ \\
\hline Count of adult diseases by CDS (SE) & $3.59(2.48)$ & $2.80(2.35)$ & $<0.01$ \\
\hline
\end{tabular}

CDS: Chronic Disease Score, a comorbidity index weighted for 29 diseases based upon pharmacy claims data 
Table 2. Unadjusted Rates of Generic Drug Utilization by Therapeutic Class among Subjects Ages 67-79 $(n=117,970)$

\begin{tabular}{|c|c|c|c|c|}
\hline & \multicolumn{2}{|c|}{$\begin{array}{l}\text { Medicare Part D } \\
\text { enrollees }\end{array}$} & \multicolumn{2}{|c|}{ Non-enrollees } \\
\hline & $2005, \%$ & $2006, \%$ & $2005, \%$ & $2006, \%$ \\
\hline \multicolumn{5}{|l|}{ Commonly used drugs } \\
\hline Antihyperlipidemics & 15.7 & 26.8 & 12.0 & 21.7 \\
\hline Antidepresssants & 60.8 & 63.9 & 57.7 & 60.8 \\
\hline Antiulcerants & 37.1 & 39.7 & 35.5 & 37.1 \\
\hline NSAIDS & 63.2 & 70.1 & 56.7 & 65.5 \\
\hline Antihistamines & 50.7 & 66.7 & 34.0 & 57.4 \\
\hline Antidiabetic drugs & 55.8 & 60.5 & 54.4 & 58.6 \\
\hline Calcium channel blockers & 50.7 & 50.7 & 48.7 & 49.2 \\
\hline ACE inhibitors & 80.4 & 80.2 & 76.2 & 75.2 \\
\hline Beta-blockers & 64.6 & 61.6 & 61.9 & 60.2 \\
\hline \multicolumn{5}{|l|}{ Special interest drugs } \\
\hline Barbiturates & 93.7 & 94.6 & 93.9 & 96.2 \\
\hline Benzodiazepines & 97.0 & 98.0 & 94.2 & 95.5 \\
\hline Antipsychotics & 35.1 & 34.1 & 40.3 & 39.7 \\
\hline Anticonvulsants & 75.3 & 70.8 & 70.5 & 64.2 \\
\hline Antivirals & 36.6 & 39.7 & 30.1 & 34.0 \\
\hline Antineoplastics & 63.2 & 56.3 & 61.3 & 58.0 \\
\hline All drugs & 57.9 & 59.5 & 54.7 & 57.4 \\
\hline
\end{tabular}

$A C E$ inhibitor $=$ angiotensin-converting enzyme inhibitor; NSAID = nonsteroidal anti-inflammatory drug

direction of change in generic drug use by Part D enrollees and non-enrollees overall and by examined therapeutic classes.

\section{Change in Utilization of Generic Drugs After Accounting for Secular Trends}

Table 3 depicts the change in utilization of generic drugs that occurred between 2005 and 2006 among beneficiaries after adjusting for beneficiaries' age, gender, English language preference, socio-demographic characteristics measured through Census zip code, 29 comorbid conditions, out-ofpocket payout ratio, the number of pharmacy claims, and 2005 Medicaid eligibility. From 2005 to 2006, there was a secular trend of increased generic drug utilization in 13 out 15 drug classes. Overall, the unadjusted odds ratio for generic drug use across all classes was 1.12 [95\% confidence interval (CI) 1.12-1.13]. However, after adjusting for the baseline differences between Part D enrollees and non-enrollees, secular trends, and other potentially confounding covariates, there was an increased use of generic drugs in two drug classes [angiotensin-converting enzyme inhibitors (OR 1.10, CI 1.051.14) and benzodiazepines (OR 1.19, CI 1.04-1.35)] and a decreased use of generic drugs in four other drug classes [antihyperlipidemics (OR 0.95, CI 0.92-0.98), nonsteroidal anti-inflammatories (OR 0.92, CI 0.87-0.97), antihistamines (OR 0.75, CI 0.70-0.82), and beta-blockers (OR 0.97, CI 0.940.99)]. Overall, beneficiaries were slightly less likely to fill prescriptions for generic drugs vs. brand-name drugs in 2006 compared to 2005 (OR 0.95, CI 0.94-0.95).

\section{Savings Associated with Greater Generic Use}

Using the same set of covariates developed in this study, generic drug use as a predictor, and total payments including third-party and patients' out-of-pocket payments as dependent variable, we estimated that on average, each generic prescription that was dispensed in 2006 saved consumers and insurers a combined total of $\$ 119.25$ annually (95\% confidence interval \$118.97-\$119.53, $\mathrm{p}<0.001)$.

\section{DISCUSSION}

In this study of a heterogeneous group of beneficiaries reflecting both Part D enrollees and non-enrollees alike, we found there was a secular trend of increased utilization of prescription

Table 3. Change in Generic Utilization by Drug Class for Subjects Aged 67-79 from 2005 to 2006

\begin{tabular}{|c|c|c|c|}
\hline \multirow[t]{2}{*}{ Drug class } & \multirow{2}{*}{$\frac{\text { Medicare Part D enrollees }}{\text { Odds ratio }(95 \% \mathrm{Cl})}$} & \multirow{2}{*}{$\frac{\text { Non-enrollees }}{\text { Odds ratio }(95 \% \mathrm{Cl})}$} & \multirow{2}{*}{$\frac{\text { Part D*Time }}{\text { Odds ratio }(95 \% \mathrm{Cl})}$} \\
\hline & & & \\
\hline Antihyperlipidemics & $1.41^{* * *}(1.37,1.45)$ & $1.97 * * *(1.93,2.01)$ & $0.95^{* * *}(0.92,0.98)$ \\
\hline Antidepresssants & $1.10^{* * *}(1.07,1.13)$ & $1.21^{* * *}(1.19,1.25)$ & $1.00(0.96,1.03)$ \\
\hline Antiulcerants & $1.04 * *(1.01,1.07)$ & $0.95^{* * *}(0.93,0.98)$ & $1.02(0.98,1.06)$ \\
\hline NSAIDs & $1.21^{* * *}(1.16,1.25)$ & $1.27 * * *(1.23,1.32)$ & $0.92^{* * *}(0.87,0.97)$ \\
\hline Antihistamines & $1.57^{* * *}(1.48,1.67)$ & $2.78^{* * *}(2.66,2.91)$ & $0.75^{* * *}(0.70,0.82)$ \\
\hline Antidiabetic drugs (excludes insulin) & $1.02 *(1.00,1.05)$ & $1.13^{* * *}(1.11,1.16)$ & $1.00(0.97,1.03)$ \\
\hline Calcium channel blockers & $1.18^{* * *}(1.15,1.21)$ & $1.06 * * *(1.04,1.09)$ & $0.98(0.94,1.01)$ \\
\hline ACE inhibitors & $1.31^{* * *}(1.27,1.34)$ & $1.09 * * *(1.07,1.12)$ & $1.10^{* * *}(1.05,1.14)$ \\
\hline Beta-blockers & $1.13^{* * *}(1.11,1.16)$ & $1.04 * * *(1.02,1.06)$ & $0.97^{*}(0.94,0.99)$ \\
\hline \multicolumn{4}{|l|}{ Special interest drugs } \\
\hline Barbiturates & - & - & - \\
\hline Benzodiazepines & $1.96^{* * *}(1.80,2.14)$ & $1.27^{* * *}(1.19,1.36)$ & $1.19^{* *}(1.04,1.35)$ \\
\hline Antipsychotics & $0.93(0.85,1.02)$ & $1.21^{* * *}(1.11,1.32)$ & $0.91(0.80,1.02)$ \\
\hline Anticonvulsants & $1.18^{* * *}(1.12,1.24)$ & $0.70^{* * *}(0.67,0.73)$ & $1.07 *(1.00,1.14)$ \\
\hline Antivirals & $1.32^{* * *}(1.15,1.50)$ & $1.32 * * *(1.15,1.50)$ & $0.99(0.83,1.19)$ \\
\hline Antineoplastics & $0.95(0.87,1.04)$ & $1.53^{* * *}(1.42,1.65)$ & $0.90(0.80,1.01)$ \\
\hline All drugs & $1.08^{* * *}(1.08,1.09)$ & $1.12^{* * *}(1.12,1.13)$ & $0.95^{* * *}(0.94,0.95)$ \\
\hline
\end{tabular}

${ }^{=}$Part D*Time represents the net effect of Part D on enrollees compared with non-enrollees after adjusting for the time trend and other potential confounders. Estimates derived from a differences-in-differences regression, adjusting for beneficiaries' age, gender, English language preference, sociodemographic characteristics measured through Census zip code, 29 comorbid conditions, out-of-pocket payout ratio, the number of pharmacy claims, and Medicaid eligibility in the base year; changes in barbiturate use could not be estimated due to the small number of observations; ACE inhibitor = angiotensin-converting enzyme inhibitor; NSAID = non-steroidal anti-inflammatory drug; ${ }^{*} P<0.05,{ }^{* *} P<=0.01,{ }^{* * *} P<=0.001$ 
of generic drugs over time for both Part D enrollees and nonenrollees. However, after adjusting for beneficiaries' demographic characteristics, baseline prescription utilization and coverage, and baseline comorbid conditions, the growth rate of generic drug use was lower among Part D enrollees than among non-enrollees, suggesting that the net effect of Part D appeared to be a decreased use of generic drugs, although the effect size and direction varied across the drug classes examined.

These results may not be reflective of all Medicare beneficiaries, and we are not able to examine the degree to which generic uptake may have differed based on specific drug plans. However, our subjects reflect a broad sample of seniors that accounts for $15 \%$ of the market share of prescription drugs in the United States. In analyses comparing our subjects to those of a nationally representative sample, our subjects were more likely to be urban dwelling, but otherwise were similar to a nationally representative sample ${ }^{6}$.

Our findings are important because little is known about the impact that Part D has had on prescription utilization, and it is unclear a priori whether or not Part D would lead to an aggregate increased or decreased rate of generic drug use. Prior work suggests that the overall impact of Part D was a modest increase in prescription utilization and decrease in out-of-pocket costs. However, we are not aware of prior peerreviewed reports examining the impact of Part D on generic drug use. Our findings are noteworthy given that generic drugs represent an important means of cost savings ${ }^{8,9}$. This is especially important given the burden of out-of-pocket costs for many Americans ${ }^{26}$, associations between cost sharing and non-adherence ${ }^{27}$, and concern about the impact of the Part D doughnut hole on processes and outcomes of care ${ }^{15,28}$.

Our inquiry is also important because the impact of Part D on generic drug use has implications for the overall impact of the benefit on prescription expenditures. Substitution towards more expensive branded drugs would be expected to lead to greater total expenditures, and greater out-of-pocket expenditures to Medicare beneficiaries, relative to what the Part D benefit would have induced had no substitution occurred (i.e., if the Part $\mathrm{D}$ benefit increased utilization of generics and branded drugs in equal proportion). To the extent that the clinical benefits of generic and branded drugs are similar, then any substitution towards branded drugs (and additional costs associated with the substitution after taking into account rebates and discounts that plans may negotiate) is wasteful. As we highlight, although the odds of generic drug use only decreased slightly as a result of Part D, given the millions of prescriptions that were examined, there may be significant economic consequences for patients and third party payers.

In our primary analyses, we adopted a patient perspective by comparing Part D enrollees with non-enrollees. Although we adjusted for several subject characteristics (e.g., sex) that might confound the associations of interest, analyses that use matching of subjects based on baseline characteristics might also be helpful to explore the impact of Part D on generic drug use. Also, in addition to comparing enrollees with nonenrollees, we examined an alternative, societal perspective by comparing Part D enrollees and non-enrollees with nearelderly subjects not yet eligible for the prescription drug benefit. These analyses yielded similar results, although of modestly lower magnitude, with an overall statistically significant decreased likelihood of generic drug use due to Part D.
There are several potential reasons why our findings differ from prior reports that suggested higher rates of generic drug use associated with Part D enrollment ${ }^{13,14}$. First, our study controlled for secular trends in generic drug use. There was a clear secular trend in increased use of generic drugs; however, our estimation suggested that such secular trend was not attributable to the institution of the Part D program. In addition, unlike those studies, we limited our analyses to a large, stable population of beneficiaries filling prescriptions for common chronic conditions during 2005 and 2006. This is important because studies that are not limited to a large stable population may erroneously attribute observed changes in rates of prescription use to changes among the users, rather than recognizing that the changes may be due to the populations being examined. In addition, while prior reports compared Part D enrollees with those with private third-party insurance, we compared Part D enrollees with the entirety of their non-enrollee counterparts within our sample.

Our findings have several limitations that lead to important yet unanswered questions. First, although we used subjects' zip code-linked Census-level data and an out-of-pocket payout ratio to reflect the financial risk and generosity of drug plans, we were unable to identify subjects' individual-level socioeconomic characteristics or details of their benefit design (e.g., premiums, copayments, PDP vs. MAPD plan), and thus we could not adjust for these variables in our analyses. Such information would be of interest since the benefit design of the drug plans often affects prescription drug consumption ${ }^{25}$, and the impact of Part D enrollment on generic drug use may differ among subjects in PDPs vs. MAPDs. Future studies with greater details of patients' economic resources and drug plans may identify the degree to which Part D has had a differential impact for subjects based on their socioeconomic strata, economic incentives such as benefit design, and enrollment in PDPs vs. MAPDs. Second, we do not have information regarding specific policies that may have been present in the pharmacy chain we examined, although we do not have any a priori reason to believe that the associations we describe would differ based on analyses of users of other pharmacy chains. Third, to maximize internal validity, our primary analyses were limited to subjects who filled at least one prescription within the pharmacy chain during both years of observation. Fourth, we did not have information regarding the negotiations that commonly take place between Part D plans and pharmaceutical manufacturers; these negotiations likely contribute to the patterns of prescription drug utilization that we describe.

In conclusion, few studies have been performed that examine the impact of the Medicare Part D Prescription Drug Benefit. Although the benefit has led to significant restructuring of pharmaceutical financing, as well as improved access for many beneficiaries, our results suggest that the institution of the Part D program led to a net decrease in the use of generic drugs among Part D enrollees compared to non-enrollees, suggesting substantial economic savings have been foregone. Future research on the economic impact of Part D should consider these opportunity costs.

Acknowledgements: Dr. Zhang was supported in part by a grant from Merck, Dr. Yin is a Robert Wood Johnson Foundation Health Policy Scholar, and Dr. Alexander has career development awards from the Agency for Healthcare Research and Quality (K08 
HS15699-01A1) and the Robert Wood Johnson Physician Faculty Scholars Program. The funding sources had no role in the design and conduct of the study, analysis or interpretation of the data, and preparation or final approval of the manuscript prior to publication.

Conflict of Interest: Dr. Zhang was supported in part by a pilot grant from the University of Chicago Center for Pharmaceutical Policy funded by the Merck Foundation. Dr. Sun previously worked at Walgreens Health Initiatives, is currently employed by Takeda Pharmaceuticals, and has been funded for other projects by grants from Merck, Novartis, BMS, and Sanofi-Aventis. Dr. Alexander has been funded for other projects by the University of Chicago Center for Pharmaceutical Policy funded by the Merck Foundation and by Pfizer.

Corresponding Author: G. Caleb Alexander, MD, MS; Department of Pharmacy Practice, University of Illinois at Chicago School of Pharmacy, Chicago, IL, USA (e-mail: galexand@uchicago.edu).

\section{REFERENCES}

1. Doherty RB. Assessing the new Medicare prescription drug law. Ann Intern Med. 2004;141:391-5.

2. Heiss F, McFadden D, Winter $\mathbf{J}$. Who failed to enroll in Medicare Part D, and why? Early results. Health Affairs. 2006;25:w344-54.

3. Cubanski J, Neuman P. Status report on Medicare Part D enrollment in 2006: analysis of plan-specific market share and coverage. Health Aff. 2006;26:w1-12.

4. Center for Medicaid and Medicare Services Press Release. Medicare drug plans strong and growing: beneficiaries compared plans and continued to sign up for prescription drug coverage. January 30, 2007. http://www. cms.hhs.gov/apps/media/press releases.asp (Accessed July 7, 2008).

5. Pauly Mv. Medicare drug coverage and moral hazard. Health Aff. 2004;23:113-22

6. Yin W, Basu A, Zhang J, Rabbani A, Meltzer Do, Alexander GC. The impact of the Medicare Part D prescription drug benefit on drug utilization and out-of-pocket costs. Annals of Internal Medicine. 2008;148:169-77.

7. Generic drug utilization in the Medicare Part D program. Department of Health and Human Services. Office of the Inspector General. November 2007 (OEI-05-07-00130).

8. Frank RG. The ongoing regulation of generic drugs. N Engl J Med 2007;357:1993-6.

9. Hass JS, Phillips KA, Gerstenberger EP, Seger AC. Potential savings from substituting generic drugs for brand-name drugs: Medical Expenditure Panel Survey, 1997-2000. Ann Intern Med. 2005;142:891-7.

10. National Association of Chain Drug Stores. Available online at http:// www.nacds.org/wmspage.cfm?parm $1=507$ (Accessed on July 7, 2008).
11. Himmel W, Simmenroth-Nayda A, Niebling W, et al. What do primary care patients think about generic drugs? International Journal of Clinical Pharmacology and Therapeutics. 2005;43:472-9.

12. Bhat VN. Patent term extension strategies in the pharmaceutical industry. Pharmaceuticals Policy and Law. 2005;6:109-22.

13. Center for Medicaid and Medicare Services Press Release. Generic drug use on the rise: consumers and payers benefit as more Americans turn to generics as way to save money and improve their health. February 8 , 2007. http://www.cms.hhs.gov/apps/media/press_ releases.asp (Accessed July 7, 2008)

14. "Medicare drug plans prompt rise in generic use." ACP Observer. 2007;27:5.

15. "Strategies to avoid Medicare's big hole." New York Times. November 24 2007

16. Poisal JA, Truffer C, Smith S, et al. Health spending projection through 2016: modest changes obscure Part D's impact. Health Aff. 2007;26:w242-53.

17. Stuart B, Singhal P. The stability of Medicaid coverage for low-income dually eligible Medicare beneficiaries. Kaiser Family Foundation Policy Brief. May 2006. Publication \#7512.

18. Geronimus AT, Bound J. Use of census-based aggregate variables to proxy for socioeconomic group: Evidence from national samples. Am J Epidemiol. 1998;148:475-86.

19. Krieger N. Overcoming the absence of socioeconomic data in medical records: Validation and application of a census-based methodology. Am J Epidemiol. 1992;82:703-10.

20. Angrist J, Krueger A. "Empirical Strategies in Labor Economics," Handbook of Labor Economics, Vol. 3, ed. by A. Ashenfelter and D. Card, Amsterdam: Elsevierss; 1999.

21. Stuart B, Simoni-Wastila L, Baysac F, Shaffer T, Shea D. Coverage and use of prescription drugs in Nursing Homes: implications for the Medicare Modernization Act. Medicare Care. 2006;44:243-9.

22. Lee M, Kang $\mathbf{C}$. Identification for difference in differences with crosssection and panel data. Economics letters. 2006;92:270-6.

23. Artz MB, Hadsall RS, Schondelmeyer SW. Impact of generosity level of outpatient prescription drug coverage on prescription drug events and expenditure among older persons. American Journal of Public Health 2002;1257-63.

24. Clark DO, Von Korff M, Saunders K, Baluch WM, Simon GE. A chronic disease score with empirically derived weights. Med Care. 1995;33:78395.

25. Goldman D, Joyce GF, Escarce JJ, et al. Pharmacy benefits and the use of drugs by the chronically ill. JAMA. 2004;291:2344-50.

26. Alexander GC, Casalino LP, Meltzer DO. Patient-physician communication about out-of-pocket costs. JAMA. 2003;290:953-8

27. Braithwaite RS, Rosen AB. Linking cost-sharing to value: an unrivaled yet unrealized public health opportunity. Ann Intern Med. 2007;146:602-5

28. Steinberg $\mathbf{M}$, Jones $\mathbf{K}$. Coverage through the 'doughnut hole' grows scarcer in 2007. Families USA, November 2006 (Publication \#06-107). 